EOMmUn: Communication et organisation

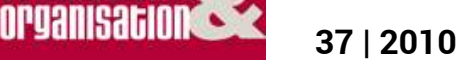

La communication à l'épreuve des mutations économiques

\title{
Une approche communicationnelle du coaching
}

\section{Geneviève Guilhaume}

\section{(2) OpenEdition \\ Journals}

Édition électronique

URL : http://journals.openedition.org/communicationorganisation/1296

DOI : 10.4000/communicationorganisation. 1296

ISSN : 1775-3546

Éditeur

Presses universitaires de Bordeaux

\section{Édition imprimée}

Date de publication : 1 juin 2010

Pagination : 115-124

ISBN : 978-2-86781-710-6

ISSN : 1168-5549

Référence électronique

Geneviève Guilhaume, "Une approche communicationnelle du coaching », Communication et organisation [En ligne], 37 | 2010, mis en ligne le 01 juin 2013, consulté le 01 mai 2019. URL : http:// journals.openedition.org/communicationorganisation/1296; DOI : 10.4000/ communicationorganisation. 1296 


\title{
Une approche communicationnelle du coaching
}

\author{
Geneviève Guilhaume'
}

Le coaching peut être appréhendé comme un dispositif destiné aux cadres qui ont la responsabilité de conduire le changement au sein d'entreprises développant des stratégies de transformation organisa-tionnelle. L'approche communicationnelle choisie interroge à la fois sa finalité dans le cadre des transformations d'entreprise requises dans un capitalisme financier et globalisé, son organisation et ses méthodes puisque ce dispositif, utilisant essentiellement des techniques de communication, doit préparer le manager d'aujourd'hui à l'ensemble de ses nouveaux rôles communicationnels, constitutifs de la mise en ouvre du changement en entreprise. Cette approche a le mérite de faire ressortir les paradoxes du dispositif. Dans quelle mesure ce dispositif ne serait-il pas alors un outil de gestion symbolique faisant intérioriser aux managers les contradictions de leurs rôles?

L'approche proposée s'appuie sur les travaux d'une thèse intitulée «Les dispositifs de coaching et de formation expérientielle destinés au managercommunicateur, enjeux communicationnels et violence symbolique en entreprise ", repris dans un livre ${ }^{2}$. L'argumentation s'appuie sur deux types de corpus: d'une part, l'étude des discours des consultants ${ }^{3}$ disponibles dans la littérature managériale portant sur le coaching, réalisée à partir des critères d'analyse dégagés dans Le nouvel esprit du capitalisme (Boltanski, Chiapello, 1999); d'autre part, l'analyse des pratiques de rôles des managers à partir des entretiens menés lors de notre enquête ${ }^{4}$.

01. Geneviève Guilhaume est maître de conférences en Sciences de l'Information et de la Communication ; elle enseigne et codirige le master professionnel Communication Organisationnelle (Communication Publique et Politique) à l'Université Bordeaux 3 (UFR Sciences des territoires et de la Communication). Elle est chercheuse et membre du MICA (axe Communication des Organisations, Université Bordeaux 3). Mail : genevieve.guilhaume@free.fr

02 . Voir référence en fin d'article

03. Nous ne mobilisons que peu ces discours de consultants, dans le cadre de cet article qui se veut synthétique, car nous privilégions les paroles de managers, issues de notre enquête, montrant l'écart existant entre la rhétorique managériale et les pratiques des rôles communicationnels. L'étude de ces discours fait l'objet des deux premiers chapitres de notre thèse et a constitué le point de départ de notre problématique. 04. L'enquête a été menée selon la méthode des entretiens semi-directifs d'une durée de trois heures environ. L'échantillon a été composé selon les critères suivants : les consultants : quatre exercent en tant 


\section{Coaching et approche communicationnelle}

Les consultants interviewés expriment bien la finalité du coaching: il convient, face à de nouveaux enjeux, de changer les attitudes et comportements du manager pour le transformer en manager-coache: «Dans la logique du manager-coache, il y a une logique d'échange, de management participatif. La deuxième notion, ce sont des attitudes de conseil, consulting vis-à-vis des collaborateurs... Une troisième notion concerne aussi le changement de rapport au pouvoir, une prise de recul, une neutralité, de l'empathie. Le manager doit faire en sorte d'aider le développement de ses collaborateurs » (entretien Rul, consultant-coache). «Le coaching, c'est l'accompagnement à l'intégration de ce qui a été révélé en quelque sorte, soit sous forme d'une nouvelle compétence soit sous forme d'un nouveau comportement » (entretien Bism, consultant-coache).

Ces nouvelles compétences attendues du manager s'avèrent être essentiellement communicationnelles. Vincent Lenhardt, un des premiers consultants à avoir écrit sur le coaching, montre que la démarche est tournée notamment vers «le développement de l'identité relationnelle, la capacité du manager à communiquer et méta-communiquer» (Lenhardt, 1992, pp. 3543). Si le manager se transforme lui-même, il devient un "porteur de sens » (Lenhardt, 1992, p. 106) pour impliquer les salariés dans les processus de changement.

Quelles sont les évolutions conduisant l'entreprise à requérir ces nouvelles capacités communicationnelles développées dans le coaching? La recherche d'une meilleure performance, face aux incertitudes renforcées d'une économie globalisée où la concurrence et la fluidité des mouvements de capitaux accroissent les risques, semble passer par le développement de capacités et d'un savoir être communicationnels du manager, forgeant en permanence son aptitude à conduire le changement. Le coaching, nous expliquent les consultants, est une pratique destinée au développement du manager lui permettant de faire face aux turbulences des économies, comme "repérer ses marges de manœuvre au sein d'enjeux flous, voire contradictoires, convaincre des nécessaires prises de risques» (Gautier, Vervisch, 2000, p. 24).

que coaches externes rattachés à un cabinet de conseil ou de façon indépendante; l'un des consultants intervient comme conseiller interne dans une entreprise, rattachée à un groupe, comme prescripteur de coaching. Les managers sont tous en position intermédiaire entre la direction générale de l'entreprise et la hiérarchie de proximité : six managers ont réalisé un coaching. Sur les managers non participants, l'un souhaite prescrire du coaching, l'autre a été prescripteur de coaching dans son poste antérieur, les deux autres font du coaching interne. Le secteur d'activité ou la taille de l'entreprise ne constituent pas un facteur discriminant. Nous avons dans notre échantillon de managers surtout de grands groupes du secteur privé, deux entreprises publiques en voie de privatisation, et deuX PME. L'âge des personnes interrogées n'a pas compté. La position dans le haut de la pyramide hiérarchique montre des âges pour la plupart compris entre quarante et cinquante ans. Les anciennetés dans l'entreprise n'ont pas joué un facteur discriminant : la plupart des managers interrogés ont une ancienneté supérieure à dix ans dans l'entreprise ou dans le groupe. 
Philippe Zarifian a bien montré, à partir de l'analyse du travail dans l'industrie sidérurgique, que c'est la nature même du travail industriel confronté à l'imprévu, qui implique la communication d'intercompréhension 5 . Notre enquête a, cependant, mis en valeur les difficultés de généraliser à l'ensemble des entreprises le modèle décrit par Zarifian, à partir des industries à flux continu; de même, les rôles communicationnels des managers ne privilégient pas toujours une communication d'intercompréhension. Ainsi, nous pouvons penser que les capacités et le savoir être communicationnels des managers sont développés dans le coaching dans un contexte où «le cadre taylorien se conjugue avec de nouvelles formes de mobilisation subjective des salariés» (Linhart, 2004).

L'approche communicationnelle du coaching met ainsi en valeur l'importance des rôles communicationnels du manager aujourd'hui. L'homme connexionniste ${ }^{6}$ d'aujourd'hui n'est plus un centre nerveux $^{7}$ dans le circuit des informations, mais au contraire un passeur, un connecteur, au mieux un point de passage obligé temporaire dans le réseau. L'autorité du manager-communicateur lui permettant de conduire les transformations organisationnelles résulte alors de ses qualités personnelles associées à son expérience, et non de sa position hiérarchique ou de son statut.

Que propose le coaching pour renforcer ce savoir être communicationnel des managers? Le coache propose un vécu expérientiel (individuel ${ }^{8}$ ) dans le dispositif, et pour agir sur le savoir être, il inscrit au cœur de la relation de face à face avec le coaché les attitudes bien connues en communication interpersonnelle de l'écoute ou du feed-back, il recourt à certaines techniques de communication, utilisées en thérapies comportementales, comme la programmation neuro-linguistique ou l'analyse transactionnelle. Ces «technologies de la communication et du changement » sont elles-mêmes légitimées par des références théoriques ${ }^{9}$.

Cette approche du coaching permet de le distinguer des dispositifs de formation plus classique, car sa finalité et ses méthodes visent l'apprentissage non de savoirs et de savoir-faire mais la transformation des attitudes et comportements. Celle-ci renvoie à deux notions différentes du savoir être ${ }^{10}$, sur lesquelles le dispositif cherche à agir:

05. «Communiquer, c'est établir des accords sur la nature des problèmes à traiter et des savoirs à développer, l'identité des objectifs et donc le processus commun de définition de ces objectifs, le sens donné aux actions, et donc les valeurs qui les fondent, les implications subjectives croisées des individus qui agissent ensemble, et donc la convergence des mobiles de ces individus » (Zarifian, 1998).

06. En référence au corpus du néomanagement du nouvel esprit du capitalisme (Boltanski, Chiapello, 1999)

07. Nous faisons là référence à l'analyse de Henry Mintzberg (Mintzberg, 1984)

08. Nous ne traitons pas le coaching d'équipe dans cet article

09. Richard Bandler, mathématicien et John Grinder, linguiste, s'appuient sur les apports de la linguistique, de la psychologie cognitive, de la neurologie, de la cybernétique et de l'informatique pour inventer la PNL. C'est le psychanalyste Éric Berne qui a modélisé la psyché en trois états du « Moi », pour analyser les transactions qui s'opèrent dans les relations interpersonnelles.

10. Nous faisons là référence aux travaux de Sandra Bellier (Bellier, 2004) 
- D'une part, le savoir être est analysé en termes de qualités morales, d'aptitudes ou de traits de caractère qu'il s'agit de déceler par des outils psychotechniques ou de faire resurgir dans une relation proche de la psychothérapie, pour sélectionner les managers les plus conformes aux normes de management de l'entreprise.

- D'autre part, il est conçu comme résultant d'un possible « apprentissage » de nouveaux comportements, à travers la notion d'expérience. De quoi s'agitil? Deux conceptions sont en présence: celle de l'expérience vécue dans la relation de coaching, au sens psychosociologique, celle du cadre expérimental, des mises en situation du dispositif de coaching renvoyant à une approche plus béhavioriste de l'acquisition de nouveaux comportements.

Le coaching est donc différent des formations plus classiques. Un autre apport essentiel de l'enquête distingue également ce dispositif, en mettant en valeur une certaine ambivalence au niveau de ses finalités, de son organisation et des méthodes utilisées. Pourquoi l'entreprise développe-t-elle alors ce type de démarche auprès des managers?

\section{Un dispositif ambivalent}

On observe des contrastes dans les finalités du coaching énoncées dans les discours des consultants-coaches sur leurs pratiques: entre la finalité vaste du développement de la personne et l'objectif étroit de la performance économique. M. Bism, consultant-coache et membre actif de l'ICF France (International Coaching Fédération) distingue le contrat officiel et le contrat caché: « le contrat officiel, c'est celui qu'on peut afficher dans l'institution, ça passe chez l'agent comptable... le contrat caché, c'est celui qu'on passe avec le coaché dans le cadre qui a été défini mais qui est beaucoup plus précis, correspondant plus à ses attentes, mais qui ne peut pas être écrit. Parce que s'il était écrit, il virerait la règle de confidentialité. Donc, ça peut être « mon chef, il a raison, mais... ce qui est mon problème c'est que je n'arrive pas à gérer mon chef » ". L'efficacité du coaching repose sur ce paradoxe, qui consiste à contourner la règle officielle, pour parvenir à transformer attitudes et comportements du coaché, en traitant les problèmes authentiques de la personne et dans le même temps à répondre à la demande du commanditaire par la restitution.

Les règles de restitution sont très strictes. « Le principe de la confidentialité est intangible », nous explique M. Rul, consultant-coache. Durée, rythme et lieux jalonnant la démarche sont définis précisément au départ, dans le contrat de coaching. Dans ce cadre, "la liberté du coache et du coaché, nous dit Mme Hisl, consultante-coache, est totale». À travers le mélange de liberté et de contraintes fortes, la relation de coaching entre le consultant, le commanditaire et le coaché apparaît elle aussi paradoxale.

On observe également une opposition entre la faible professionnalisation des coaches et les cahiers des charges drastiques des entreprises. En effet, il 
n'existe pas de référentiel standard ${ }^{11}$ des compétences attendues des coaches, même si les formations au coaching se développent dans le secteur privé et public. Les méthodes et les références théoriques sont variables selon les coaches. Les managers interrogés reconnaissent que le coaching est proche de la démarche thérapeutique, mais s'en éloigne aussi, car il ne s'exerce que dans un cadre professionnel. Certaines méthodes sont très personnelles: M. Bism vante les mérites de la méthode de l'aïkido, appliquée à la démarche. L'appartenance à une fédération de coaching proposant aux coaches des certifications ne garantit pas pour autant la déontologie de la profession. Le consultant explique: "Donc, c'est pas une organisation professionnelle dans le sens syndicat, c'est pas non plus un lieu de garantie de supervision et de validation des coaches... c'est juste un regroupement de coaches dont la valeur est très, très, très relative, très honnêtement... c'est-à-dire que pour être à la SFC [Société Française de Coaching], il faut payer, comme pour être à l'ICF [International Coaching Federation], il suffit de payer ». Les professionnels interrogés mettent tous en avant l'importance de l'éthique personnelle du coache, se substituant à la déontologie. En contrepartie, ce sont les entreprises elles-mêmes "qui définissent ces référentiels de compétences, dans le cadre de cahiers des charges très précis » (entretien Mel, consultant interne à la Direction du Conseil en Management du Groupe Automobile).

Ces paradoxes s'exercent facilement, car l'évaluation du dispositif et de ses effets reste floue. Les consultants interviewés confirment qu'ils travaillent dans le cadre « d'un engagement de moyens mais pas de résultats ». Pourquoi l'entreprise a-t-elle recours à ce dispositif ambivalent, dont les résultats ne peuvent être évalués? Que nous apporte l'enquête sur ce point? Elle montre de fortes contradictions vécues par les managers dans leurs pratiques communicationnelles.

\section{Des rôles communicationnels contradictoires}

Précisons que les contradictions de rôle analysées ici sont en fait le plus souvent des paradoxes: des oppositions entre des termes, des objets (ici des discours ou des pratiques portant sur la communication) faisant intervenir des types et des niveaux logiques distincts. Les injonctions paradoxales ou double binds sont celles où le méta-message contredit le message (Barel, 1999). Dans le discours du néo-management (Boltanski, Chiapello, 1999) par exemple, le facilitateur " redistribue les informations dans les équipes, dans les réseaux. C'est un impulseur de vie, de sens, d'autonomie ». Il sait aussi prendre des risques pour se connecter, être un pilleur d'idées, à la façon du chercheur d'or, pour développer les connexions riches de potentialités nouvelles ». D'un côté, il crée une relation de confiance, ce qui renvoie à la logique de l'organisation (dans les réseaux): c'est le méta-message. De l'autre, il développe des qualités lui permettant de «s'ajuster aux circonstances » comme le demande la logique 11. Chaque association professionnelle propose le sien 
du marché : c'est le message; Méta-message et message peuvent alors entrer en contradiction, formant un paradoxe, dans cette confusion des sphères.

Ces contradictions sont analysées comme des processus, qui révèlent les contradictions du système avec lui-même (Barel, 1999): en effet, l'entreprise a besoin de la rationalisation, du contrôle, de l'imposition des règles, de la contrainte (hiérarchique). Elle a besoin de la libre parole, des «parlers ordinaires» permettant l'appropriation (Mispelblom, 1999), de la communication d'intercompréhension, permettant la coopération dans le travail (Zarifian, 1999). Comment ces contradictions analysées comme des paradoxes s'inscrivent-elles dans les pratiques?

Prenons l'exemple du rôle de mobilisateur: Mme Adre, conseillère ressources humaines pour la Direction des Technologies et Systèmes d'Information dans le Groupe Automobile évoque son rôle "d'animation des équipes » (des généralistes ressources humaines positionnés en unités opérationnelles): « Donc, moi,j’en ai quand même dix à animer, plus trois en direct, dans le sens d'un déploiement des projets RH. Cette animation, c'est faire en sorte qu'ils appliquent la politique ressources humaine de GA, qu'ils déploient, quand on a des projets spécifiques ressources humaines. Par exemple, pour DTSI, depuis l'année dernière, on a remis en place toute la démarche compétence et nomenclature des métiers ». Il s'agit bien d'une communication, qui sous-couvert d'une démarche participative vise le déploiement de projets: "c'est s'assurer que les choses sont faites dans les délais ». La responsable évoque également la communication dans la conduite d'un changement organisationnel décidé par la direction: elle consiste à « vendre » la nouvelle organisation (du travail) aux salariés: «Moi, je n'en sais rien si l'organisation $\mathrm{A}$ ou $\mathrm{B}$ était bonne. Je veux dire, à la limite, ce n'est pas mon souci. Moi, mon souci, c'était de vendre l'organisation B, c'était de faire en sorte que les managers comprennent pourquoi la A n'était pas bonne, la B était bonne, et pourquoi il fallait fonctionner dans l'organisation B ».

Cet exemple illustre une communication de mobilisation qui ne vise pas l'implication par l'appel à une parole autonome et intercompréhensive des managers de proximité, mais qui a pour but de déployer des transformations organisationnelles, d'imposer des réorganisations. Ce processus contradictoire, nous le retrouvons dans tous les rôles communicationnels des managers de notre enquête: il exprime la tension entre ancien et nouveau système (le modèle du cadre taylorien et celui du manager-communicateur). Il traduit aussi l'opposition entre deux conceptions de la performance: à moyen terme, la compétence exige une communication authentique et intercompréhensive des salariés, constitutive d'autonomie et de coopération. À court terme, dans le cadre du capitalisme financier et des réorganisations incessantes qu'il implique, les managers imposent souvent une communication dogmatique ${ }^{12}$ (du sommet ou à travers les dispositifs participatifs).

12. Au sens où l'entend B. Floris lorsqu'il évoque la doxa (Floris, 2e semestre 2000), au sens de N. Alter lorsqu'il analyse les inventions dogmatiques (Alter, 2000). 
Ce conflit entre deux conceptions de la performance est bien illustré par l'exemple suivant d'un manager souhaitant mobiliser ses hiérarchiques de proximité. M. Guit, responsable de la communication et des ressources humaines du site et responsable des relations sociales de la filiale du Groupe Chimie évoque l'organisation de réunions participatives pour soutenir les hiérarchiques de proximité, qui ont pour rôle de mobiliser les ouvriers dans une usine au fonctionnement taylorien, où l'autonomie laissée aux exécutants reste très faible, où la réduction d'effectifs liée à la mondialisation entraîne une large démotivation de la base et la réaction combative d'un syndicat puissant. Plus implicitement, ces rencontres de libre parole permettent de redonner du pouvoir aux managers de proximité par rapport au syndicat, mais plus modestement de leur faire supporter leur positionnement, "de vider leur sac sur les difficultés qu'ils ont ». Là, le dispositif participatif vise bien à susciter une communication authentique des ingénieurs chefs d'atelier, non pas pour accroître leurs performances dans la conduite du changement (rôle du mobilisateur), mais pour les aider dans leur rôle de silentblok, d'amortisseur des «secousses", pris entre les revendications de la base et les orientations stratégiques du sommet, dans un contexte de concurrence exacerbée et de restructuration.

Ces paradoxes dans les pratiques communicationnelles ne sont-ils pas alors le vecteur de nouvelles formes de violence symbolique s'imposant par et sur les managers? Nous évoquons le terme de violence, car les contradictions de rôle que nous analysons se traduisent par de véritables injonctions paradoxales: "Linjonction paradoxale barre la possibilité même du choix, rien n'est possible, et une suite alternée infinie est alors déclenchée » (Watzlawick, Helmick-Beavin, Jackson, 1972). N'est-ce pas une communication paradoxale que celle évoquée par M. Deleb, secrétaire général du centre d'ingénierie du Groupe Energie qui vise à rendre les salariés « acteurs " d'une réorganisation des fonctions tertiaires se traduisant pour eux par des conséquences difficiles en termes de vie au travail ou de mode de vie?

Cette communication paradoxale agità travers un processus d'euphémisation des discours. Nous faisons là référence au concept défini par Pierre Bourdieu: l'euphémisation consiste en un « travail de dissimulation et de transfiguration qui assure une véritable transsubstantiation des rapports de force en faisant méconnaître-reconnaître la violence qu'ils enferment objectivement et en les transformant ainsi en pouvoir symbolique capable de produire des effets réels sans dépense apparente d'énergie » (Bourdieu, 1982). Les paroles citées du manager sont ainsi significatives : évoquer le terme « d'acteurs » pour les salariés s'inscrit dans un discours managérial sur l'autonomie, la responsabilisation, alors que la réalité est celle d'une restructuration de l'entreprise imposée.

Les managers interrogés ont une forte conscience de ces contradictions de leurs rôles communicationnels, ils révèlent aussi une souffrance au travail: M. Lain, responsable de la stratégie d'implantation tertiaire (Groupe 
Automobile), raconte: « dans le coaching, ily a eu une phase qui était appliquée à ma vision des choses, ma problématique, mon angoisse professionnelle éventuellement, et ça a basculé à mi-parcours, une fois que ça a été épuisé, que j'accepte ça, et je tire un trait là-dessus, sur « comment je prends la suite ", c'est ça mon problème; c'est comment je crée une division opérationnelle avec quatre services qui tirent dans quatre directions différentes, tout en motivant les gens, tout en leur donnant du rêve et de la vision, tout en écartant ceux qui ne sont pas récupérables ". Le "ça ", évoqué dans le verbatim renvoie à l'acceptation d'un management paradoxal, consistant à impliquer les salariés dans la réorganisation, tout en en renvoyant certains, mission que le coaching lui permet de réaliser, au prix d'une souffrance s'exprimant par "l'angoisse professionnelle ». Le coaching semble donc jouer un rôle dans l'intériorisation de ces paradoxes.

\section{Le coaching pour faire intérioriser aux managers les contradictions de leurs rôles}

Notre hypothèse est que le vécu de la relation de coaching, constituée de liberté et de contraintes prépare aux contradictions des rôles communicationnels. M. Kell, Directeur d'établissement de vente de véhicules et pièces de rechange et atelier de réparation (Groupe Automobile) a parfaitement intégré l'ambivalence requise dans le style de communication du manager: "Le coaching permet de prendre conscience des... je dirai des inconvénients que telle ou telle attitude peut avoir. Et c'est clair que tout comportement est ambivalent. Quand je dis que j’ai le goût du rapport de force, il faut évidemment veiller à ce que cette tendance-là ne débouche pas sur un management brutal. Donc, encore une fois, le coaching, c'est comment utiliser son tempérament et sa personnalité propre sans jamais faire que cette personnalité propre devienne un handicap ».

L'intériorisation des paradoxes est d'autant plus forte que les effets du coaching sont avérés: la plupart des managers coachés reconnaissent un «effet miroir » impliquant du coaching, c'est-à-dire l'accès à une meilleure connaissance de soi, dans le but de développer les capacités managériales. On observe aussi un effet de prise de conscience, qui agit sur les représentations. M. Lain,Directeurdelastratégied'implantationtertiaire(Groupe Automobile) explique les effets pour lui du coaching: "Avant, je faisais mon métier plus du management, alors que j’ai inversé la chose maintenant, je bascule dans une préoccupation de conduite et d'animation d'équipe ». Les effets du coaching sont avant tout personnels: les managers interviewés prennent conscience de leur savoir être préparant à une communication différente ensuite avec les équipes. Une emprise élevée du dispositif de coaching sur les managers montrerait son rôle dans l'exercice de nouvelles formes de domination passant par des pratiques communicationnelles paradoxales. Notre enquête montre que cette emprise est effective pour les managers qui ont été sélectionnés pour des mobilités promotionnelles ou qui souhaitent l'être. Les stratégies 
personnelles d'utilisation de ce dispositif existent également (cas du coaching servant à quitter l'entreprise). Il existe enfin un coaching de remédiation aidant les managers à supporter une souffrance au travail, liée aux paradoxes de leurs rôles. Nous pouvons donc avancer que cette approche communicationnelle du coaching permet de le distinguer des autres dispositifs de formation des managers, en montrant l'importance de la communication constitutive des actions de changement conduites par ces responsables. Elle met en valeur un dispositif ambivalent participant à l'émergence d'une violence symbolique. Les managers la portent, mais aussi la subissent ou développent des logiques d'action à son encontre.

\section{BIBLIOGRAPHI}

\section{Documents-sources}

BARANSKI L.,Lemanageréclairé:piloterle changement, Paris, Éditions d'Organisation, 2001

KERJEAN A., Les nouveaux comportements dans l'entreprise: oser secouer l'organigramme, Paris, Éditions d'Organisation, 2000, (Collection Tendances)

GAUTIER B., VERVISCH M-O., Le manager coache, Paris, Dunod, 2000

LENHARDT V., Les responsables porteurs de sens: culture et pratique du coaching et du team-building, Paris, INSEP Consulting Éditions, 1992

LE SAGET M., Le manager intuitif, Paris, Dunod, 1998

Ouvrages ou articles scientifiques

BAREL Y., Le paradoxe et le système, Grenoble: PUG, 1989

BELLIER S., Le savoir être dans l'entreprise: utilité en gestion des ressources humaines, Paris, Vuibert, 2004, (Collection Entreprendre Vital Roux)

BOLTANSKI L., CHIAPELLO È., Le nouvel esprit du capitalisme, Paris, Gallimard, 1999, (Collection NRF Essais)

BOURDIEU P., Langage et pouvoir symbolique, Éditions du Seuil, 2001, (Collection Points)

FLORIS B., DE CRESCENZO J-C., «La gestion participative comme objet de communications organisationnelles ", in DELCAMBRE P. (coord.), Communications organisationnelles: objets, pratiques, dispositifs, Rennes, Presses Universitaires de Rennes, $2^{\mathrm{e}}$ semestre 2000, pp. 159-174

GUILHAUME G., L'ère du coaching, critique d'une violence euphémisée, Paris, Syllepse, 2009

LINHART D., La modernisation des entreprises, La Découverte, 2004, (Collection Repères)

MINTZBERG H., Les dix rôles du cadre, Paris, Éditions d'Organisation, 1984

MISPELBLOM-BEYER F., "Langages au travail et normes d'assurance qualité », Sociologie du Travail, 1999, vol. 41, n 3 , pp. 235-254 
SEGRESTIN D., Les chantiers du manager: l'innovation en entreprise, où en sommesnous? Comment piloter les changements et les maîtriser?, Paris, Armand Colin, 2004, (Collection Sociétales)

WATZLAWICK P., HELMICK-BEAVIN J., JACKSON DON D., Une logique de la communication, Paris : Éditions du seuil, 1972, (Collection Points)

ZARIFIAN P., Travail et communication, Paris, PUF, 1998, (Collection Sociologie d'aujourd'hui)

Résumé : L'approche communicationnelle du coaching permet de mettre en valeur ses finalités, méthodes et références théoriques visant le développement d'un savoir-être communicationnel des managers, devenant central dans la conduite du changement des entreprises dans un contexte de concurrence mondialisée et du capitalisme financier. Elle fait ressortir l'ambivalence du dispositif dans les pratiques, contribuant à faire intérioriser par les managers les paradoxes de leurs rôles communicationnels: ceux du facilitateur ou du mobilisateur par exemple. À travers cette communication paradoxale émergent de nouvelles formes de violence symbolique portées et supportées par les managers. L'emprise du coaching sur les responsables est avérée. Ceux-ci ont, cependant, une forte conscience des contradictions à l'œuvre dans le dispositif et dans leurs pratiques managériales, expriment une souffrance au travail ou développent des logiques d'action plus personnelles dans ce cadre.

Mots-clés : coaching, savoir être, communication paradoxale, contradictions, euphémisation, violence symbolique, intériorisation.

\begin{abstract}
The communication approach of coaching enables to bighlight its final aims, methods and theoretical references aiming at developing the communicational knowledge of managers, becoming essential in leading the reform of firms in a context of world-wide competition and financial capitalism. It brings out the ambivalence of the system in place in the practice, contributing to have the managers internalize the paradoxes of their communicational roles: for instance, those of the facilitator and the mobilizor. Through this paradoxal communication emerge new forms of symbolic violence borne and endured by managers. The coaching ascendancy over the managers is recognized. They have, nevertheless, a strong awareness of the contradictions at play in the system and in their managerial practices; they express a suffering at work or develop more personal actions in this context.
\end{abstract}

Keywords : coaching, appropriate managerial behavior, paradoxal communication, contradictions, euphemismed language, symbolic violence, internalization. 